\title{
INFLUÊNCIA DA OTITE MÉDIA NO TRANSTORNO FONOLÓGICO: ANÁLISE ACÚSTICA DA DURAÇÃO DAS FRICATIVAS DO PORTUGUÊS BRASILEIRO
}

\author{
Otitis media influence on phonological disorders: \\ an acoustic analysis for duration of Brazilian Portuguese fricatives
}

\author{
Haydée Fiszbein Wertzner ${ }^{(1)}$, Luciana de Oliveira Pagan ${ }^{(2)}$, Adriana Limongeli Gurgueira ${ }^{(3)}$
}

\begin{abstract}
RESUMO
Objetivo: verificar a duração da produção de fricativas surdas e sonoras ensurdecidas em crianças com e sem histórico de otite média (OM). Métodos: 12 crianças (seis com e seis sem transtorno fonológico) sendo três com o processo de ensurdecimento de fricativas e $\mathrm{OM}$, três com ensurdecimento sem OM, três sem ensurdecimento com OM e três sem ensurdecimento sem OM, provenientes do LIF em Fonologia do Departamento de Fisioterapia, Fonoaudiologia e Terapia Ocupacional da Faculdade de Medicina da USP-SP. Foram apresentadas as figuras correspondentes às palavras ['fila]x['vila], ['cinco]x['zinco], ['xis]x['giz] para nomeação. Os dados foram coletados e armazenados diretamente no Computerized Speech Laboratory (4300B). Resultados: o grupo sem TF e sem OM produziu os sons sonoros com duração menor que os surdos (diferença estatisticamente significante), assim como o grupo com OM, porém sem significância estatística. Os dois grupos com ensurdecimento, com e sem OM, não apresentaram diferença de duração na produção dos sons. Conclusão: a OM influencia a percepção de diferenças na duração entre os sons fricativos surdos e sonoros, apesar da duração não ser a única pista acústica para o contraste da sonoridade.
\end{abstract}

DESCRITORES: Transtornos do Desenvolvimento da Linguagem; Otite Média; Desenvolvimento Infantil; Acústica da Fala

\section{INTRODUÇÃO}

Dentre as alterações de linguagem e fala detectadas em crianças, uma das mais ocorrentes é o transtorno fonológico ${ }^{1,2}$. O transtorno fonológico pode ser definido como uma alteração caracterizada pela produção inadequada dos sons da fala relacionada ao uso impróprio das regras fonológicas da língua, às dificuldades na articulação dos sons ou de percepção dos sons.

(1) Fonoaudióloga; Professora Associada Livre do curso de Fonoaudiologia da Faculdade de Medicina da Universidade de São Paulo, FMUSP, São Paulo, SP.

(2) Fonoaudióloga clínica; Doutora pela Faculdade de Filosofia Letras e Ciências Humanas da Universidade de São Paulo.

(3) Fonoaudióloga; Professora da Faculdade de Ciências Médicas da Santa Casa de São Paulo, FCMSC-SP, São Paulo, SP; Doutora pela Faculdade de Filosofia Letras e Ciências Humanas da Universidade de São Paulo.
Para a descrição linguística das alterações fonológicas encontradas nos sujeitos com transtorno fonológico é necessária a escolha de um modelo teórico. Essa análise pode ser realizada por meio do modelo de processos fonológicos, em que se verificam quais as regras fonológicas da língua que foram simplificadas. O processo fonológico é definido como uma simplificação aplicada a uma classe de sons ${ }^{3,4}$.

No português brasileiro os processos de ensurdecimento de plosivas e fricativas, caracterizados pela ausência do traço distintivo de sonoridade, estão entre os mais observados em sujeitos com transtorno fonológico ${ }^{5,6}$. Tal processo tem grande importância uma vez que muitas crianças passam a realizar essa alteração na leitura e na escrita a partir da alfabetização ${ }^{7}$.

Muito embora os processos de ensurdecimento final e sonorização inicial ocorram durante o desenvolvimento fonológico de crianças falantes do inglês, 
no português tais processos foram pouco observados no desenvolvimento normal de crianças brasileiras a partir de 3:1 anos ${ }^{8}$. Estudos sobre o uso de processos fonológicos em crianças normais 9,10 mostraram que o ensurdecimento não foi produtivo entre 2:0 e 3:0 anos. Em crianças com transtorno fonológico ${ }^{11}$, verificou-se que a faixa etária em que o maior número de sujeitos apresentou unicamente o processo de ensurdecimento foi entre 6:0 a 9:0 anos.

Vários fatores de risco têm sido estudados como variáveis de interferência para alterações do desenvolvimento fonológico (nível sócio-econômico, percepção auditiva, linguagem, inteligência e desenvolvimento psicossocial entre outros) ${ }^{12}$.

O papel da percepção auditiva tem sido enfatizado como essencial no desenvolvimento e na construção do inventário fonético da criança.

Apesar de ser possível a identificação do transtorno fonológico, ainda não se consegue determinar uma única causa. Dentre as diferentes etiologias, uma delas está relacionada a episódios de OM de repetição ${ }^{13}$.

Em recentes estudos ${ }^{13-16}$ observa-se a crescente preocupação no estabelecimento de possíveis marcadores diagnósticos que possam ajudar na identificação e no tratamento precoce de crianças com transtorno fonológico.

A OM é uma infecção caracterizada por uma inflamação na orelha média frequentemente associada a um aumento de fluido infectado, ou não, de grande prevalência na primeira infância e que decresce com a idade ${ }^{17}$. Estudos apontam que $50 \%$ das crianças com um ano de idade apresentaram pelo menos um episódio de otite, sendo que entre um e três anos de idade $35 \%$ destas crianças apresentarão otites de repetição ${ }^{18}$.

Muitos estudos vêm sendo realizados para determinar a influência da OM nas alterações de fala e linguagem. Alguns não acharam diferenças significantes na produção articulatória entre crianças que apresentaram problemas de OM na infância e crianças sem queixas auditivas ${ }^{19,20}$. Outros, entretanto, relatam que crianças com o histórico de otite apresentaram desempenho mais baixo em testes de percepção e processamento auditivo além de grande incidência de problemas acadêmicos, especialmente alterações de leitura e escrita ${ }^{1,21}$.

Os pesquisadores que acreditam que a OM não tem efeito sobre a linguagem argumentam que a perda auditiva condutiva varia de leve a moderada (perda auditiva flutuante) durante os episódios de otite. Assim, após estas ocorrências, a audição volta à normalidade e os efeitos da otite são compensados pela audição normal. Já aqueles que acreditam que a OM pode trazer consequências para o desenvolvimento fonológico, defendem que a perda auditiva transitória causada pela otite pode resultar num sinal auditivo incompleto ou inconsistente, levando a uma fraca codificação das distinções fonéticas ${ }^{22}$. A perda auditiva causada pela otite faz com que alguns sons tornem-se inaudíveis, alterando a percepção e/ou a produção da fala ${ }^{23}$.

A análise acústica tem se mostrado bastante útil para auxiliar no diagnóstico na medida em que estuda as propriedades acústicas dos sons da fala, que são fundamentais na aquisição e desenvolvimento desses sons ${ }^{11,24,25}$. Este tipo de análise permite verificar as variações de intensidade, tempo e frequência dos sons da fala.

Estudos recentes sugerem que os ouvintes são sensíveis aos detalhes fonéticos e retêm na memória muitas, se não todas, as variantes fonéticas de uma palavra. Esses detalhes fonéticos (características acústicas dos sons) podem dar ao ouvinte informações cruciais sobre a percepção que ele tem dos sons ${ }^{26}$.

Em se tratando da sonoridade, uma série de parâmetros acústicos tem sido identificada para sua distinção em consoantes fricativas: presença de periodicidade, intensidade e duração do ruído fricativo, padrões de frequência fundamental das vogais anteriores ou posteriores ao fonema fricativo e a duração da transição do formante da própria vogal ${ }^{27}$.

Um estudo preliminar com fricativas para verificar o uso da duração como pista para o contraste de sonoridade mostrou que as fricativas surdas apresentaram-se mais longas que as fricativas sonoras ${ }^{28}$. Apesar dessa diferença, quando analisado o uso da duração como pista distintiva do traço de sonoridade, os achados da pesquisa sugeriram que a duração do ruído de fricção não poderia ser considerada um atributo primário para a distinção entre fricativas surdas e sonoras. Os mesmos resultados foram encontrados em estudo semelhante ${ }^{29}$. Quando o ruído de fricção é relativamente de longa duração, as fricativas tendem a ser percebida como surdas ${ }^{30}$.

Considerando que o processo de ensurdecimento tem relação com a percepção dos sons da fala e voltando-se para a identificação de um subtipo para o transtorno fonológico, o objetivo deste estudo foi verificar acusticamente a duração das consoantes fricativas surdas e sonoras ensurdecidas produzidas por crianças com TF separados em dois grupos, com e sem histórico de OM, comparando os resultados desta medida aos de crianças sem TF igualmente separadas em um grupo com e outro sem OM. 


\section{MÉTODOS}

Trata-se de um estudo descritivo, transversal e quantitativo. Todos os pais ou responsáveis pelos sujeitos assinaram o Termo de Consentimento Pós-Informação.

Participaram desta pesquisa 12 crianças, seis com o processo de ensurdecimento de fricativas (com TF) e seis sem alterações fonológicas (sem TF), com idades entre 6:0 e 9:0 anos.

As crianças com transtorno fonológico foram provenientes do Laboratório de Investigação Fonoaudiológica (LIF) em Fonologia do Departamento de Fisioterapia, Fonoaudiologia e Terapia Ocupacional da Faculdade de Medicina da Universidade de São Paulo. Esses sujeitos foram reunidos de acordo com o histórico de otite em dois grupos: sem histórico de OM (GE1) e com histórico de OM (GE2).

Todos os sujeitos realizaram processo diagnóstico no referido LIF entre novembro de 2004 e maio de 2005 onde foi confirmado o transtorno fonológico.

Os critérios de inclusão foram: o uso do processo fonológico de ensurdecimento de fricativas caracterizado pela substituição dos fonemas fricativos sonoros pelos seus cognatos surdos, ausência de alteração auditiva, idade entre 6:0 e 9:0 anos, não estarem em processo terapêutico por mais de um mês.

As crianças sem TF foram selecionadas de uma escola privada da região do Butantã, sendo submetidas à avaliação da fonologia, do vocabulário, do discurso e da audição. Os critérios de inclusão foram ausência de qualquer déficit auditivo e/ou alteração fonológica, idade entre 6:0 e 9:0 anos e não ter realizado nenhum tipo de tratamento fonoaudiológico em qualquer época da vida. Os sujeitos sem transtorno fonológico também foram agrupados de acordo com o histórico de otite, constituindo os grupos GC1 sem histórico de otite e GC2 com histórico de otite. Para o diagnóstico do transtorno fonológico foram aplicados os testes de Fonologia, Vocabulário, Fluência e Pragmática do ABFW-Teste de Linguagem Infantil ${ }^{31}$, as provas de percepção auditiva, consciência fonológica, elaboração de discurso a partir de estímulo visual e avaliação do sistema estomatognático aplicadas no LIF de fonologia.

Todos os sujeitos realizaram audiometria e imitanciometria no Setor de Audiologia do Centro de Docência e Pesquisa de Fisioterapia, Fonoaudiologia e Terapia Ocupacional da Faculdade de Medicina da Universidade de São Paulo na qual foi confirmada a ausência de alterações auditivas no período da coleta.

A coleta de dados para análise acústica foi realizada por meio da apresentação de seis figuras pareadas /fila/x/vila/, /cinco/x/zinco/, /xis/x/giz/ que representavam palavras com os pares de fonemas $/ f / x / v /, / s / x / z /, / S / x / 3 /$ em posição de onset. Os pares de figuras caracterizavam pares mínimos com oposição quanto ao traço distintivo de sonoridade. As figuras em sua maioria contemplaram palavras dissílabas sendo apenas um dos pares composto por monossílabos (xis X giz). Os estímulos a serem nomeados foram apresentados na tela de um computador por meio do Microsoft Powerpoint.

Os dados foram coletados e armazenados diretamente no CSL (Computerized Speech Laboratory fabricado pela Kay Elemetrics - modelo 4300B) com a utilização de um microfone unidirecional da marca Shure, modelo SM-58. Em seguida, as amostras de fala foram analisadas espectrograficamente no próprio CSL.

Todos os grupos foram avaliados individualmente na mesma sala pela terceira autora deste artigo. Foram necessárias quatro sessões com duração de uma hora para a avaliação de cada criança dos dois grupos.

Para a coleta da nomeação dos pares mínimos inicialmente foi verificado se as crianças conheciam todas as figuras que seriam apresentadas. As figuras não reconhecidas foram nomeadas pelo examinador para que a criança aprendesse a nomeá-las. Após a confirmação de que todas as figuras seriam nomeadas pelo sujeito, iniciava-se a testagem propriamente dita.

A criança foi colocada em frente à tela do computador. O microfone foi instalado em um pedestal e posicionado em ângulo de 45 a $90^{\circ}$ mantendo-se a distância entre 10 e $20 \mathrm{~cm}$ da boca do indivíduo.

As crianças foram solicitadas a produzir três vezes cada uma das palavras fila, vila, cinco, zinco, xis e giz a partir da apresentação de figuras em diferentes ordens. Os estímulos foram apresentados um a um, e deveriam ser nomeados após a seguinte ordem dada pela examinadora: "Vão aparecer algumas figuras no computador e eu quero que você diga o nome delas para mim, mas você só poderá falar depois que eu perguntar". As ordens dadas pela examinadora foram diferentes, variando em função dos estímulos nas figuras. Na Tabela 1 observa-se a palavra alvo e a ordem dada na apresentação de cada figura.

Para manter a fidedignidade da gravação no CSL, a taxa de amostragem (sampling rate) foi ajustada para 20.000 amostras por segundo. A análise espectrográfica foi realizada para todos os sujeitos dos dois grupos. Para tanto, cada uma das emissões foi analisada em forma de onda e em espectrografia. 
Tabela 1 - Palavra alvo e ordem dada na apresentação das figuras GE1

\begin{tabular}{cccc}
\hline Cinco & "Que número é este?" & Zinco & "Do que são feitas estas moedas?" \\
\hline Xis & "Que letra é esta?" & Giz & "O que é isto?" \\
\hline Fila & "O que é isto?" & Vila & "O que é isto?" \\
\hline
\end{tabular}

Após a obtenção da forma de onda e do espectrograma a duração dos sons alvo /f/x/v/, /s/x/z/, / $/$ $\mathrm{x} / 3$ / foi delimitada visual e auditivamente.

Para as crianças com transtorno fonológico, os dados referentes à presença ou ausência do histórico de otite foram obtidos a partir da análise dos prontuários fonoaudiológicos e, para as crianças sem transtorno fonológico, esses dados foram obtidos a partir da anamnese realizada com os pais. A presente pesquisa foi aprovada pelo Comitê de Ética em Pesquisa da Universidade de São Paulo sob o número 372/04.

Para este trabalho foram utilizados os testes não paramétricos de WILCOXON e ManN-WhitneY. Além dos testes, fez-se uso de uma técnica para complementar a análise descritiva, o INTERVALO DE Confiança. O nível de significância estabelecido para o estudo foi de 0,08 (n sig. 0,08 ) devido à baixa amostragem.

\section{RESULTADOS}

Inicialmente foram comparadas as durações entre os sons $/ \mathrm{f} / \mathrm{x} / \mathrm{v} /, \mathrm{s} / \mathrm{x} / \mathrm{z} / \mathrm{e} / \mathrm{s} / \mathrm{x} / \mathrm{z} /$ produzidos nas palavras /fila/ e /vila/, /cinco/ e /zinco/, /xis/ e /giz/ para cada um dos quatro grupos: GE1, GE2, GC1 e GC2.
Em relação ao grupo GE1, observou-se diferença estatisticamente significativa $(p=0,046)$ apenas entre as médias de xis e giz, sendo que a duração foi maior para o/3/ produzido com ensurdecimento. A Figura 1 mostra os valores médios de duração dos três pares estudados, para este grupo.

Para o grupo GE2, os resultados mostraram-se estatisticamente significantes $(p=0,069)$ somente quando as médias dos sons $/ \mathrm{f} / \mathrm{x} / \mathrm{v} /$ produzidos nas palavras /fila/ e /vila/ foram comparadas. Para este grupo, o maior valor foi observado para o som /f/, produzido na palavra /fila/, como pode ser observado na Figura 2.

Para o grupo GC1, verificou-se uma diferença média que é considerada estatisticamente significante entre Fila/Vila $(p=0,008)$ e Xis/Giz $(p=0,015)$. Para Cinco/Zinco, existe uma tendência à diferença, isso porque o $p$-valor está muito próximo do limite aceitável $(p=0,090)$. A Figura 3 mostra os valores médios das durações dos sons estudados produzidos pelo grupo GC1 nas palavras fila, vila, xis, giz, cinco e zinco.

Para o grupo GC2 foi encontrada diferença média estatisticamente significante $(p=0,051)$ apenas entre a duração dos sons/s/ e /z/ do par/cinco/ $\mathrm{X} / \mathrm{zinco}$. A Figura 4 apresenta os valores médios para este grupo para os três pares de palavras.

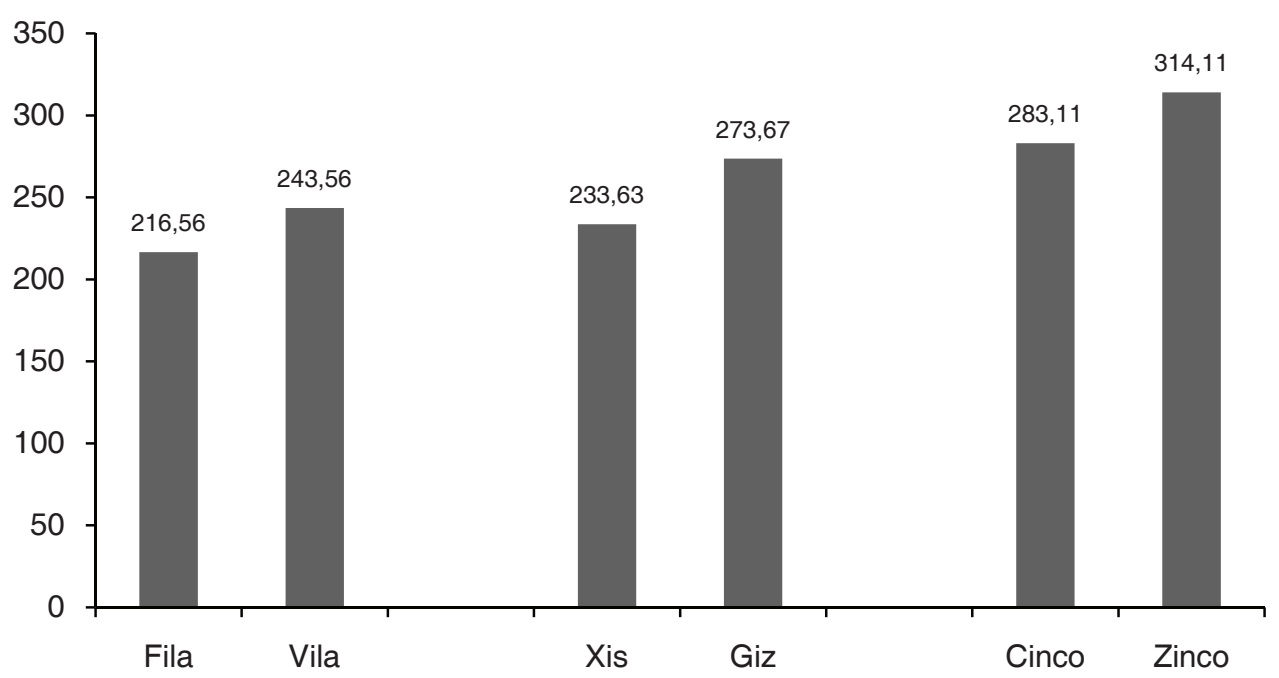

Figura 1 - Valores médios da duração dos sons /f/, /v/, /s/, /z/, /S/, /z/, para o grupo 


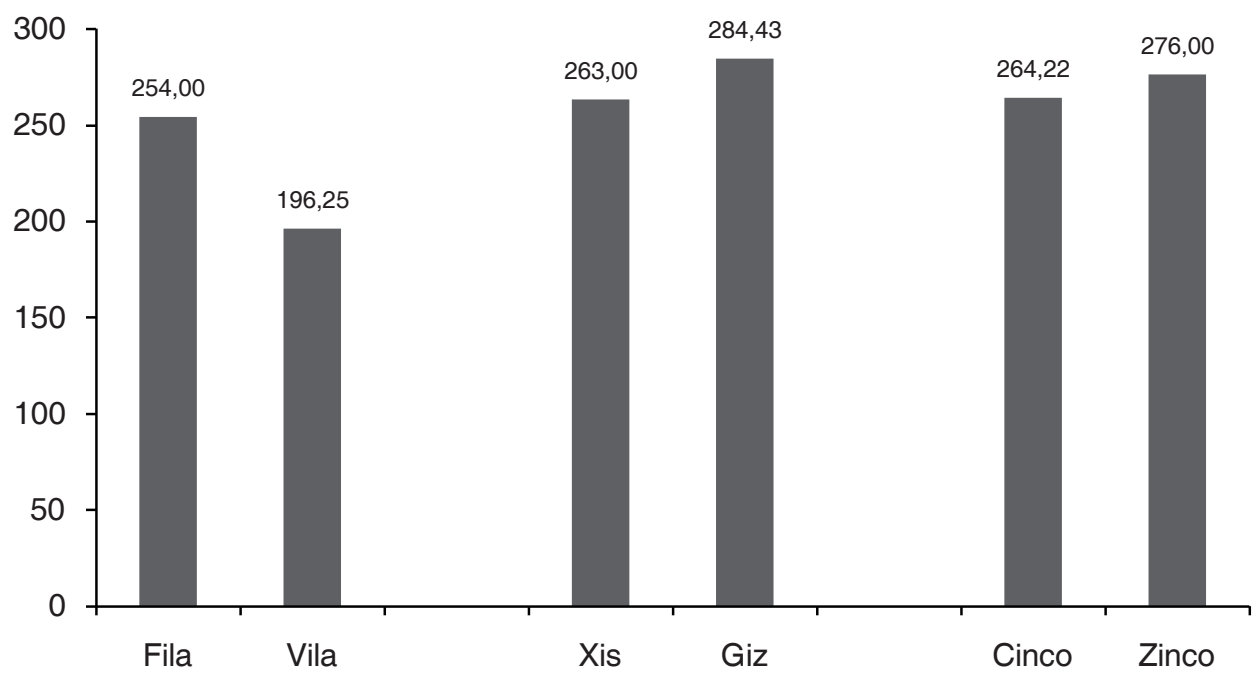

Figura 2 - Valores médios da duração dos sons /f/, /v/, /s/, /z/, / //, /3/, para o grupo GE2

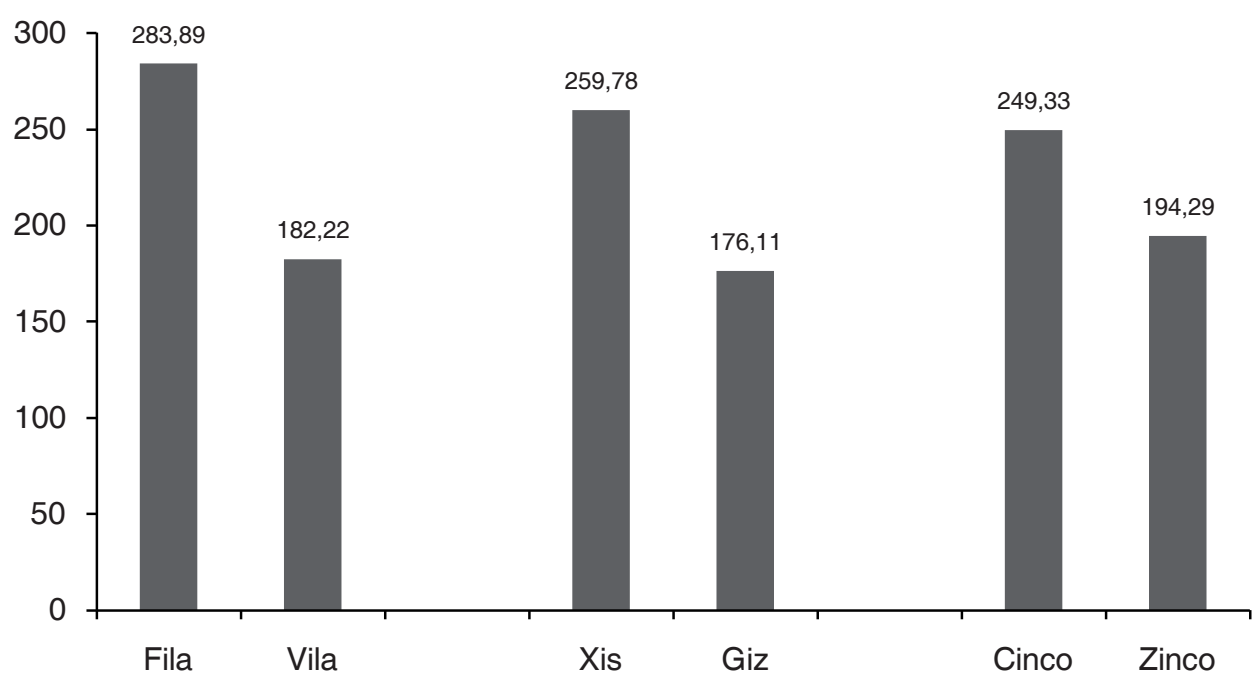

Figura 3 - Valores médios da duração dos sons /f/, /v/, /s/, /z/, /J/, /z/, para o grupo GC1

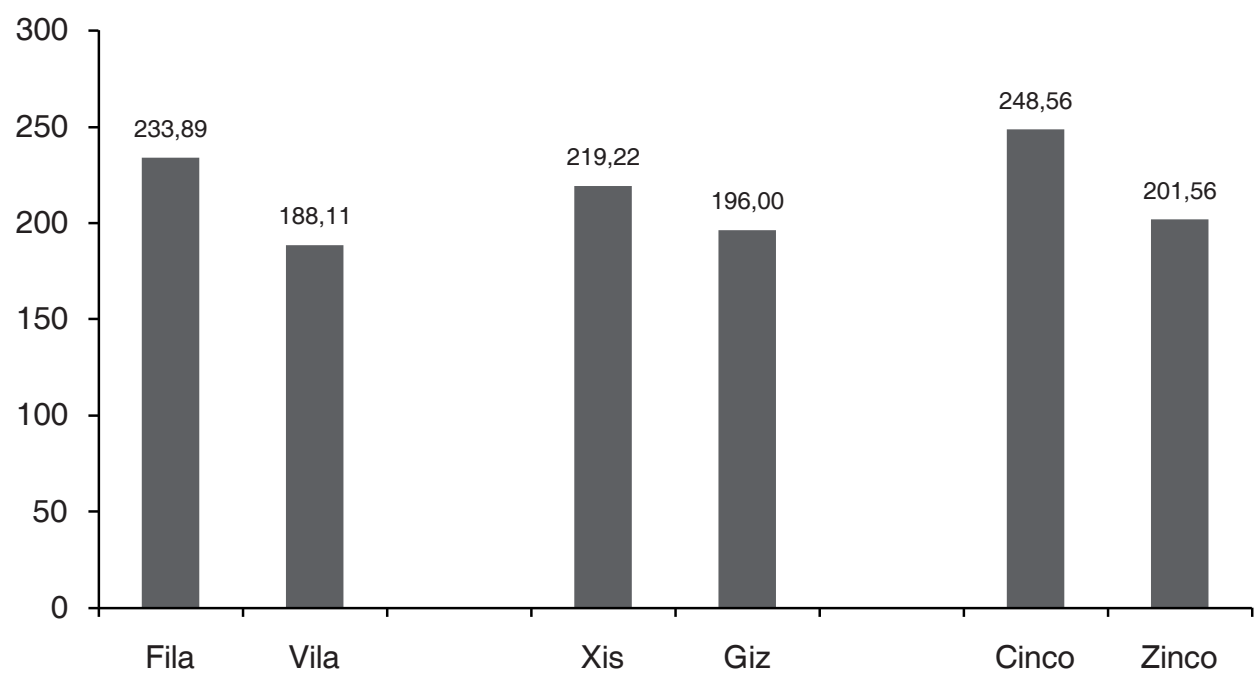

Figura 4 - Valores médios da duração dos sons /f/, /v/, /s/, /z/, /S/, /z/, para o grupo GC2 
A seguir, foram comparados tanto para o grupo com TF como para o grupo sem TF, os indivíduos com e sem otite.

Comparando-se a duração dos sons estudados entre os grupos GE1 e GE2, observou-se diferença estatisticamente significante $(p=0,054)$ apenas para o som /v/ ensurdecido, emitido na palavra /vila/. Os indivíduos com processo de ensurdecimento e sem histórico de OM apresentaram maior duração desse som. A Figura 5 mostra os valores médios da duração de cada som para os dois grupos.

$\mathrm{Na}$ análise entre os grupos GC1 e GC2 não foram encontradas, em nenhum dos sons estudados, diferenças médias estatisticamente significantes. Os valores médios para esses grupos podem ser visualizados na Figura 6.

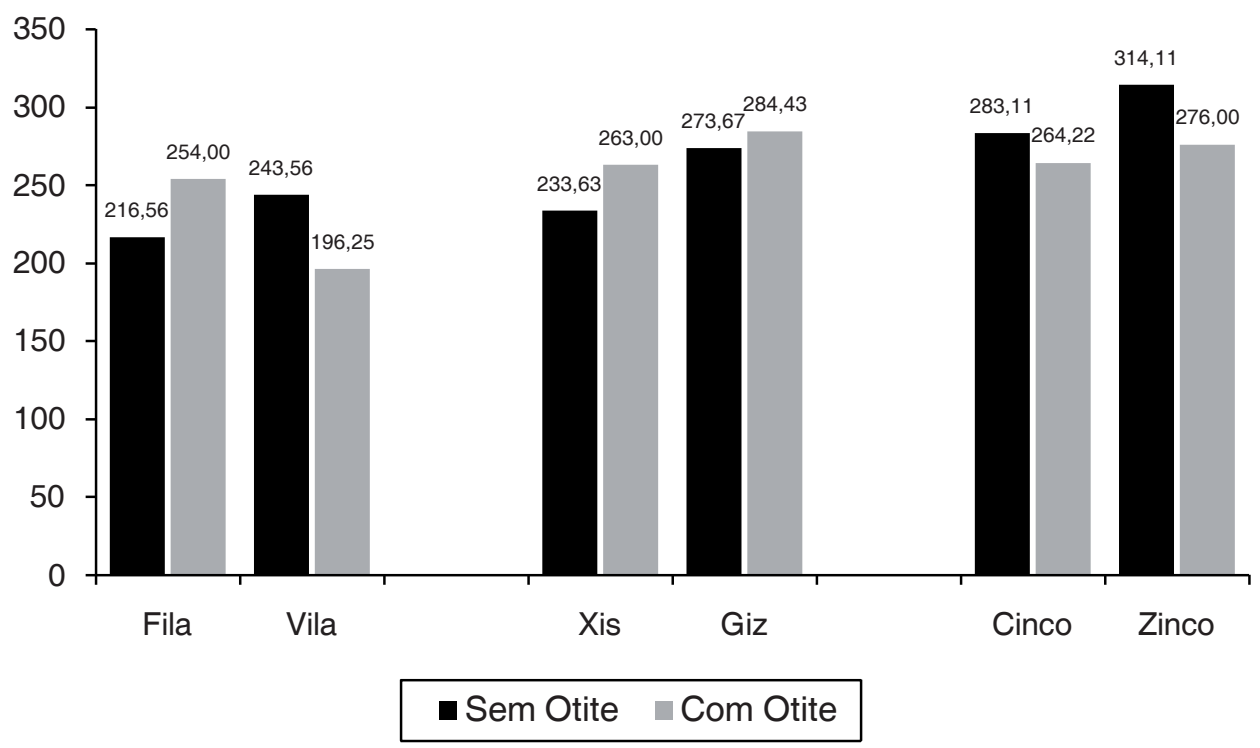

Figura 5 - Valores médios da duração dos sons /f/, /v/, /s/, /z/, /J/, /Z/, para os grupos GE1 e GE2

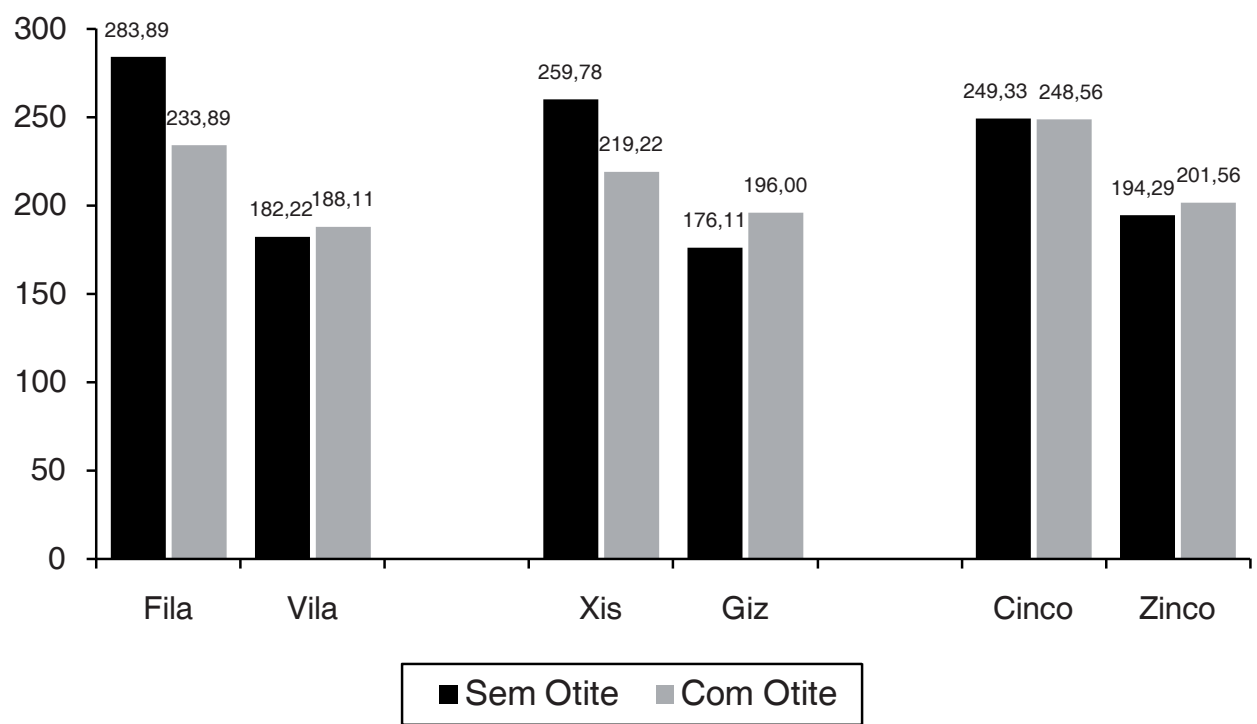

Figura 6 - Valores médios da duração dos sons /f/, /v/, /s/, Iz/, //J/, /z/, para os grupos GC1 e GC2 


\section{DISCUSSÃO}

Os resultados obtidos na análise quantitativa propiciaram algumas reflexões em relação à OM como fator causal do transtorno fonológico, bem como quanto ao uso da análise acústica, através do parâmetro de duração, na verificação de diferenças entre as produções de crianças com e sem transtorno fonológico.

Inicialmente, analisando-se os grupos das crianças com processo de ensurdecimento, os resultados sugerem que apesar de não terem tido otite, as crianças do grupo GE1 não mostraram distinção do parâmetro acústico de duração para o contraste de sonoridade e, mesmo sendo estatisticamente significante, o som /3/ ensurdecido foi produzido com maior duração que o som / / o que não era esperado, uma vez que na produção normal, os sons surdos apresentam maior duração que os sons sonoros ${ }^{28-30}$.

Estes achados quanto à diferença entre os sons /3/ ensurdecido e o som / $/$ / sugerem que as crianças do GE1 já percebiam alguma distinção de sonoridade utilizando-se de outras pistas acústicas. Estudos de variáveis acústicas permitem verificar que diferentes padrões acústicos da produção da fala (duração, frequência e intensidade) são utilizados como pistas com maior ou menor importância, em momentos distintos do desenvolvimento, sendo que mais de um aspecto pode estar envolvido na percepção de um mesmo contraste ${ }^{32-34}$.

Para o grupo GE2 (ensurdecimento e otite), resultados semelhantes foram encontrados. Não houve diferenças entre as médias dos sons surdos e sonoros para os pares $/ \mathrm{s} / \mathrm{X} / 3 /$ e $/ \mathrm{s} / \mathrm{X} / \mathrm{z} /$ como já era esperado, uma vez que os sujeitos desse grupo produziram os sons sonoros de forma ensurdecida. Para o par $/ f / X / v /$, observou-se diferença estatisticamente significante, sendo o som / $/$ / ensurdecido produzido com menor duração do que o seu cognato surdo, o que sugere que para este par, apesar das crianças desse grupo terem apresentado episódios de otite, elas estavam fazendo alguma distinção acústica mais próxima à normalidade entre os sons surdos e sonoros (sons surdos com maior duração que os sonoros) ainda que a produção do som /v/ fosse ensurdecida. Estes dados sugerem que, talvez, a ocorrência dos episódios de OM não seja suficiente para prejudicar a detecção dos detaIhes fonéticos importantes para a distinção entre os fonemas ${ }^{26}$.

Comparando-se as produções de cada som estudado entre os grupos GE1 e GE2 observou-se que tanto os sons surdos /f/, / / / e /s/ quanto os sonoros ensurdecidos /3/ e /z/ apresentaram durações semelhantes. O som $/ \mathrm{v} /$ (ensurdecido), foi o único que apresentou diferença estatisticamente significante entre os grupos, evidenciando que o grupo sem histórico de otite apresentou esse som com maior duração que o grupo com histórico de otite, ou seja, o grupo que mais se aproximou dos padrões de normalidade foi o grupo com histórico de otite.

Esses resultados podem levar a duas explicações possíveis. Por um lado, a OM pode não ser um fator que interfira na distinção entre surdas e sonoras, o que corrobora os achados de outros estudos que relacionam OM e percepção de fala ${ }^{19,20}$. Por outro, como se trata da investigação de um único parâmetro acústico (diferenças de duração) podese pensar que, talvez, este não seja o parâmetro mais importante para a distinção da sonoridade, e que a OM poderia causar consequências mais prejudiciais quanto à percepção de outros parâmetros acústicos como frequências formantes, intensidade entre outros ${ }^{21}$.

Examinando-se os grupos sem transtorno fonológico, observou-se que no grupo sem histórico de otite, os sons sonoros de todos os pares estudados, foram produzidos com duração bem menor do que os sons surdos, e essa diferença foi estatisticamente significante. Para o grupo com histórico de otite, embora os sons sonoros, tenham sido produzidos com menor duração do que seus cognatos surdos, a diferença não foi estatisticamente significante. Tais resultados mostram que para as crianças sem histórico de otite, há uma distinção mais definida entre os sons surdos e sonoros, observada nesta diferença de duração, enquanto que para o grupo com histórico de otite, essa distinção está menos marcada, o que permite evidenciar que a otite pode ter prejudicado a percepção mais acurada das diferenças de duração entre os sons surdos e sonoros, concordando com um estudo que relata que a perda auditiva transitória causada pela otite pode resultar em um sinal auditivo incompleto ou inconsistente, levando a uma fraca codificação das distinções fonéticas ${ }^{22}$.

Embora o grupo com histórico de otite tenha apresentado diferenças menores entre as durações dos fonemas surdos e sonoros, os sujeitos desse grupo não apresentavam o processo de ensurdecimento, e isso pode ser explicado pelo fato da duração não ser a única pista para a percepção do contraste de sonoridade dos sons fricativos ${ }^{27}$.

$\mathrm{Na}$ comparação das produções de cada som estudado entre os grupos GC1 e GC2, não foram encontradas diferenças estatisticamente significantes, nem na comparação entre os sons surdos, nem entre os sons sonoros. Estes dados reforçam, mais uma vez, que a duração não é a única pista acústica para a distinção da sonoridade, conforme descrito na literatura ${ }^{27}$. 


\section{CONCLUSÃO}

O estudo evidenciou que as crianças do GC1, sem otite, diferenciaram os sons surdos e sonoros por meio da duração dos sons enquanto que os demais grupos não o fizeram. A análise da duração dos sons surdos e dos sons sonoros não mostrou diferença na comparação entre GE1 e GE2 bem como entre GC1 e GC2 .
Portanto, os resultados do presente trabalho evidenciam que a otite pode prejudicar a percepção de diferenças de duração entre os sons surdos e sonoros, mas por não ser esta a única pista acústica para o contraste de sonoridade este déficit na percepção de duração, não acarretará necessariamente no uso do processo fonológico de ensurdecimento.

\section{ABSTRACT}

Purpose: acoustically analyze the duration of voiced and voiceless fricatives produced by children with and without otitis media (OM) history. Methods: 12 children (three having the phonological process of devoicing fricatives with $\mathrm{OM}$, three having devoicing without $\mathrm{OM}$, three without devoicing with $\mathrm{OM}$ and three without devoicing and without OM). Children were enrolled in a speech program at the Laboratory of Speech and Language Investigation in Phonology of the University of Sao Paulo, Brazil. They were asked to say the name of the pictures representing the words ['fila]x['vila], ['cinco]x['zinco], ['xis]x['giz]. Data were collected and stored in the Computerized Speech Laboratory (4300B). Results: the duration of voiceless fricatives was longer than the voiced fricatives for the two groups without devoicing, but there was statistical significance only for the group without OM. The two groups presenting devoicing, with and without $\mathrm{OM}$, did not show difference in the duration of the studied sounds. Conclusion: media otitis may not be the only acoustical feature that affects sound contrast, but it affects the perception of the differences in duration between the voiced and voiceless fricative sounds.

KEYWORDS: Language Development Disorders; Otitis Media; Child Development; Speech Acoustics

\section{REFERÊNCIAS}

1. Shriberg LD, Kwiatkowski J. Phonological disorders I: a diagnostic classification system. J Speech Hear Dis. 1982; 47:226-41.

2. Gierut JA. Treatment efficacy: functional phonological disorders in children. J Speech Lang Hear Res. 1998; 41:S85-S100.

3. Hodson BW, Paden EP. Phonological processes which characterize unintelligible and intelligible speech in early childhood. J Speech Hear Dis. 1981; 46(3):369-73.

4. Edwards ML. In support of phonological processes. Lang Speech Hear Serv Sch. 1992; 23:233-40.

5. Oliveira MMF, Wertzner HF. Estudo do distúrbio fonológico em crianças. Rev Soc Bras Fonoaudiol. 2000; 7:68-75.

6. Wertzner HF. O distúrbio fonológico em crianças falantes do português, descrição e medidas de sonoridade [livre-docência]. São Paulo (SP): Universidade de São Paulo; 2002.

7. Wertzner HF, Papp ACS, Amaro L, Galea DES. Relação entre processos fonológicos e classificação perceptiva de inteligibilidade de fala no transtorno fonológico. Rev Soc Bras Fonoaudiol. 2005; 10(4):193-200.

8. Wertzner HF. Articulação: aquisição do sistema fonológico dos 3 aos 7 anos [tese]. São Paulo (SP): Universidade de São Paulo; 1992.

9. Galea DES, Almeida RC, Wertzner HF. Uso dos processos fonológicos de ensurdecimento de plosivas e fricativas, plosivação de fricativas, harmonia consonantal e redução de sílaba em crianças de 2;1 a 3;0 anos de idade. IV Congresso Internacional de Fonoaudiologia e III Encontro Ibero-Americano de Fonoaudiologia, São Paulo, SP; 1999. p.50.

10. Galea DES. Análise do sistema fonológico em crianças de 2,1 a 3,0 anos de idade [dissertação]. São Paulo (SP): Universidade de São Paulo; 2003.

11. Gurgueira AL. Estudo acústico dos fonemas surdos e sonoros do português do Brasil, em crianças com distúrbio fonológico apresentando o processo fonológico de ensurdecimento [dissertação]. São Paulo (SP): Universidade de São Paulo; 2000.

12. Shriberg LD, Kwiatkowski J, Gruber FA. Developmental phonological disorders II: short-term speech- sound normalization. J Speech Hear Res. 1994; 37(5):1127-50. 
13. Shriberg LD. Epidemiologic and diagnostic profiles for five developmental phonological disorders [publicação online]. Seminar presented at the Annual Convention or the American SpeechLanguage-Hearing Association 1999. Disponível em: URL: http://www.waismain.wisc.edu/phonology 14. Shriberg LD. Classification and misclassification of child speech sound disorders. Annual Convention of the American Speech-Language-Hearing Association; 2002 Novembro; Atlanta, Georgia.

15. Shriberg LD, Kent RD, Karlsson HB, McSweeny JL, Nadler CJ, Brown RL. A diagnostic marker for speech delay associated with otitis media with effusion: backing of obstruents. Clin Linguist Phon. 2003; 17(7):529-47.

16. Shriberg LD, Flipsen Junior $P$, Kwiatkowski $\mathrm{J}$, McSweeny JL. A diagnostic marker for speech delay associated with otitis media with effusion: the intelligibility-speech gap. Clin Linguist Phon. 2003; 17(7):507-28.

17. Friel-Patti S. Otitis media with effusion and the development of language: a review of the evidence. Top Lang Dis. 1990; 11(1):11-22.

18. American Speech and Hearing Association. Otitis media, hearing and language development [publicação online]. 2000. Disponível em: URL: http:// www.asha.org/consumers/brochures/otitismedia.htm 19. Harsten G, Nettelbladt U, Schalén L, Kalm O, Prellner K. Language development in children with recurrent acute otitis media during the first three years of life. Follow-up study from birth to seven years of age. J Laringol Otol. 1993; 107:407-12.

20. Wertzner HF, Rosal CAR, Pagan LO. Ocorrência de otite média e infecções de vias aéreas superiores em crianças com distúrbio fonológico. Rev Soc Bras Fonoaudiol. 2002; 7(1):31-7.

21. Shriberg LD, Flipsen Junior $P$, Thielke $H$, Kwiatkowski J, Kertoy MK, Katcher ML, et al. Risk for speech disorder associated with early recurrent otitis media with effusion: two retrospective studies. J Speech Lang Hear Dis. 2000; 43(1):79-99.

22. Gravel JS, Wallace IF. Listening and language at 4 years of age: effects of early otitis media. J Speech Lang Hear Res. 1992; 35(3):588-95.
23. Schochat E, organizador. Processamento auditivo. São Paulo: Lovise; 1996. 142p.

24. Gurgueira AL. Estudo acústico do Voice Onset Time (VOT) e da duração da vogal na distinção da sonoridade dos sons plosivos em crianças com transtorno fonológico [tese]. São Paulo (SP): Universidade de São Paulo; 2006.

25. Pagan LO. Estudo das líquidas laterais e vibrantes em crianças com distúrbio fonológico: analise acústica e articulatória [dissertação]. São Paulo (SP): Universidade de São Paulo; 2003.

26. Nguyen N, Hawkins S. Temporal integration in the perception of speech: introduction. J Phonetics. 2003; 31(3):279-87.

27. Pirello K, Blumstein SE, Kurowisk K. The characteristics of voicing in syllable-initial fricatives in American English. J Acoust Soc Am. 1997; 101(6):3754-65.

28. Baum SR, Blumstein SE. Preliminary observations on the use of duration as a cue to syllable-initial fricative consonant voicing in English. J Acoust Soc Am. 1987; 82(3):1073-7.

29. Jongman A. Duration of frication noise required for identification of English fricatives. J Acoust Soc Am. 1989; 85(4):1718-25.

30. Borden GJ, Harris KS, Raphael LJ. Speech science primer: physiology, acoustics, and perception of speech. Baltimore: Williams \& Wilkins; 1994.

31. Andrade CRF, Befi-Lopes DM, Fernandes FDM, Wertzner HF. ABFW - Teste de linguagem infantil nas áreas de fonologia, vocabulário, fluência e pragmática. Carapicuíba: Pró-Fono; 2000.

32. Kent RD. Motor control: neurophysiology and functional development. In: Caruso A, Strand E. Clinical management of motor speech disorders in children. New York: Thieme Medical Publishers, Inc; 1999. p. 29-70.

33. Nittrouer S. Learning to perceive speech: how fricative perception changes, and how it stays the same. J Acoust Soc Am. 2002; 112(2):711-9.

34. Mayo C, Turk A. Adult-child differences in acoustic cue weighting are influenced by segmental context: children are not always perceptually biased toward transitions. J Acoust Soc Am. 2004; 115(6):3184-94.

DOI: 101590 / S1516 - 18462009005000015

RECEBIDO EM: 18/10/2007

ACEITO EM: 25/05/2008

Endereço para correspondência:

Luciana de Oliveira Pagan

Rua Dr. Nogueira Martins, 400 ap. 52

São Paulo - SP

CEP: 04143-020

E-mail: lucianapagan @ hotmail.com 